\title{
Beam Condition Monitoring with Diamonds at CDF
}

\author{
Peter Dong*, Ricardo Eusebi ${ }^{\dagger}$, Charlie Schrupp*, Anna Sfyrla ${ }^{\ddagger}$, Rick Tesarek ${ }^{\dagger}$ and Rainer Wallny* \\ *University of California, Los Angeles, Los Angeles, California 90024 \\ ${ }^{\dagger}$ Fermi National Accelerator Laboratory, Batavia, Illinois 60510 \\ $\ddagger$ University of Geneva, CH-1211 Geneva 4, Switzerland
}

\begin{abstract}
Particle physics collider experiments at the high energy frontier are being performed today and in the next decade in increasingly harsh radiation environments. While designing detector systems adequate for these conditions represents a challenge in itself, their safe operation relies heavily on fast, radiation-hard beam condition monitoring (BCM) systems to protect these expensive devices from beam accidents. The talk will present such a BCM system based on polycrystalline chemical vapor deposition ( $p C V D$ ) diamond sensors designed for the Collider Detector at Fermilab (CDF) experiment operating at Fermilab's Tevatron proton-antiproton synchrotron. We report our operational experience with this system, which was commissioned in the spring of last year. The system currently represents the largest of its kind to be operated at a hadron collider. It is similar to designs being pursued by the next generation of hadron collider experiments at the Large Hadron Collider (LHC).
\end{abstract}

\section{INTRODUCTION}

Particle physics collider experiments at the high energy frontier are being performed in increasingly harsh radiation environments. While designing detector systems adequate to these conditions represents a challenge in itself, their safe operation relies on fast, radiation-hard beam condition monitoring $(\mathrm{BCM})$ systems to protect these delicate devices from beam accidents. We present such a BCM system based on polycrystalline chemical vapor deposition (pCVD) diamond sensors used at the Collider Detector at Fermilab (CDF) experiment operating at Fermilab's Tevatron proton-antiproton synchrotron. We report our operational experience with this system, which was commissioned in the spring of 2006. The system is currently the largest of its kind to be operated at a hadron collider and it is is similar to designs being pursued by the next generation of hadron collider experiments at the Large Hadron Collider (LHC).

\section{A. Beam structure}

The Tevatron collides beams of protons and antiprotons with a center of mass energy of $\sqrt{s}=1.96 \mathrm{TeV}$. The beam structure of the Tevatron is the same for protons and antiprotons except for the direction of the beam. The Tevatron is operated with a radio frequency of $53 \mathrm{MHz}$, resulting in RF buckets of $18.8 \mathrm{~ns}$.

During Run II the Tevatron uses 36 bunches each of protons and antiprotons. The beam configuration is shown in Figure 1. The 36 bunches are distributed in three trains of 12 bunches each. The bunches in a train are separated by $21 \mathrm{RF}$ buckets (396 ns). The trains are separated by a 139-bucket (2617 ns) gap called the abort gap. If protons and antiprotons were orbiting in a central orbit (as depicted in Figure 1) collisions will occur at many points. The points marked B0, D0 and F0

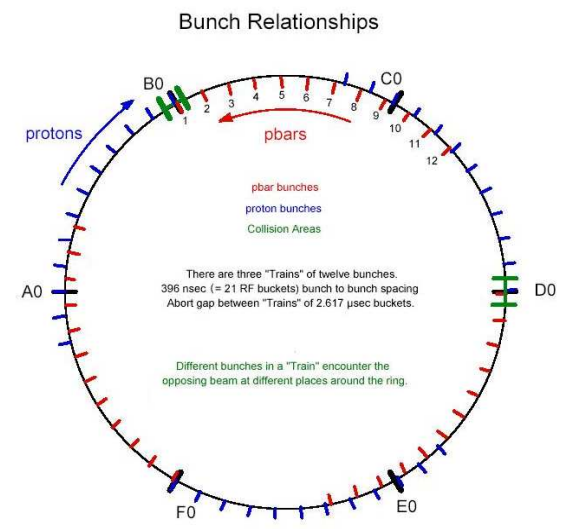

Fig. 1. Bunch structure of the Tevatron. The 36 proton bunches go clockwise and are shown as blue marks outside the ring. Antiprotons go counterclockwise and are shown as red marks inside the ring. Detectors are located at the points labeled B0 (CDF) and D0.

would have the maximum number of collisions (12) per turn. However detectors are located only at the B0 and D0 points and collisions produced at other points in the ring are wasted. To avoid this inefficiency, a set of electrostatic separators are used to create a pair of non-intersecting helical closed orbits with protons in one strand and antiprotons in the other. The helical configuration prevents the beams from colliding. This step is done after proton injection; antiprotons are then injected in their strand of the helix. Once both beams are in the Tevatron, the particles are accelerated to $980 \mathrm{GeV}$. Then the injection helix is changed to the collision helix, in which separator bumps close to the interaction points phase the helix so that the proton and antiproton beams collide only at the center of the detectors. After collimators are inserted to remove beam halo, the detectors turn on and begin taking physics data. Bunch crossings occur 12 times every $396 \mathrm{~ns}$, followed by a gap of $2.6 \mu \mathrm{sec}$, and this sequence is repeated three times in a single beam revolution. One complete revolution takes $21 \mu \mathrm{s}$.

\section{B. Beam incidents and the need for a better BCM system}

Instabilities and sudden losses in the particle beam are an unavoidable part of running a large accelerator, but pose a threat to particle detectors. Although many sub-detectors are affected in beam incidents, the largest consequences are for the CDF silicon strip detector. This detector is the closest to the beam pipe, and in past beam incidents, the large and acute radiation fields damaged many chips embedded in the 


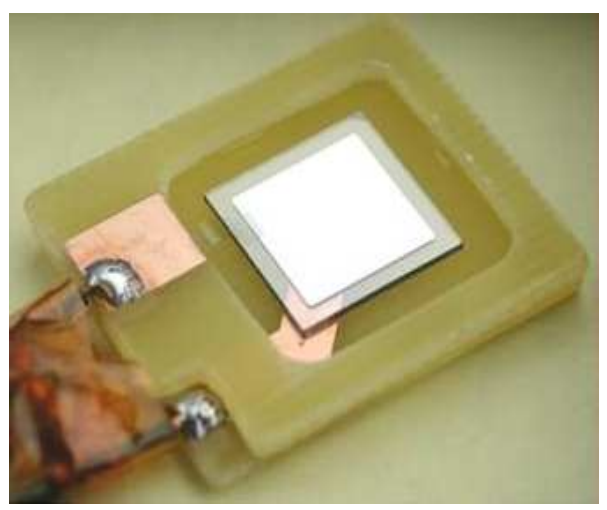

Fig. 2. A diamond sensor in a G10 package. The bias voltage is applied to the lower-right lead, which connects to the metallized back side of the sensor through a copper line. The upper-left lead is for readout and is wire-bonded to the upper metalized side of the sensor.

detector. The current BCM system consists of four Beam Loss Monitors (BLMs), two on the east and two on the west side of the detector, about $4.3 \mathrm{~m}$ from the nominal interaction point (IP) and at a radius of about $20 \mathrm{~cm}$ from the beam axis. The distance of the BLMs from the I.P. was determined completely by their size, which made it impossible to put them inside the detector. The BLMs are read out every $210 \mu$ s (10 beam revolutions), and a circular buffer of 2048 measurements is kept. Most of the incidents in which damage to the silicon detector occured showed radiation below the readout threshold before the beam-abort signal (issued by a different mechanism) and complete saturation in the next measurement. They could not resolve the beam structure well enough to forsee the abort, and gave no indication of the instability of the beam.

A closer examination of these incidents made it clear that the BCM system could be improved with smaller sensors placed closer to the beam line and to the silicon detector. Also important is a faster readout system, with which the structure of beam incidents can be studied.

\section{DIAMOND-BASED BCM SYSTEM}

Diamond sensors satisfy both these needs: they can be made as small as a square centimeter, and their signal formation time is on the order of nanoseconds. The following subsections detail the diamond-based BCM system, starting with the sensors, following with their location in the CDF detector and finishing with the readout electronics.

\section{A. Diamond sensors}

Diamonds can be used as particle detectors. When a charged particle passes through a diamond, the atoms of the lattice are ionized, passing electrons to the conduction band and leaving holes in the valence band. A minimum ionizing particle will leave, on average, 3.5 electron-hole pairs per traversed $\mu \mathrm{m}$. A voltage applied to the diamond can force the pairs to drift, generating a measurable current. Typical diamond sensors are $300-700 \mu \mathrm{m}$ thick; however, due to defects in the crystal lattice, the typical distance electron-hole pairs separate is about $250 \mu \mathrm{m}$.

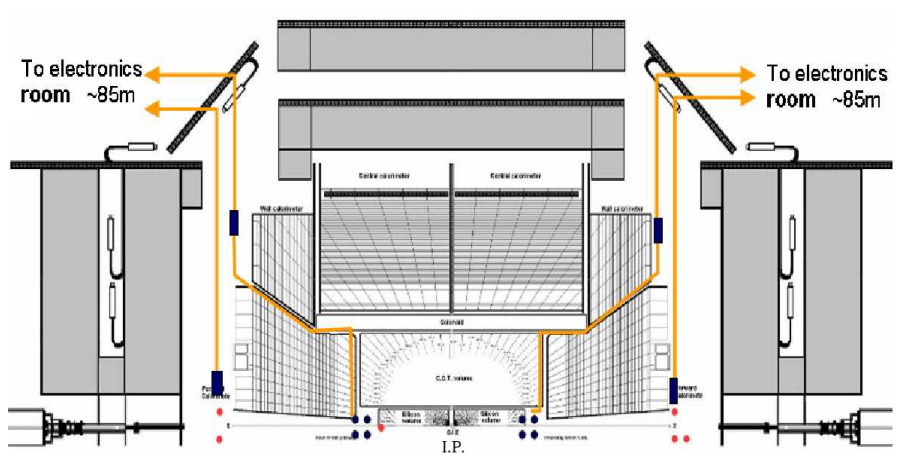

Fig. 3. The location of the diamonds in the CDF detector. The picture shows the upper hemisphere of the CDF detector. The proton beam circulates along the horizontal axis, colliding with the anti-proton beam that circulates in the opposite direction. The collision occurs at the interaction point, indicated by "I.P." in the figure. The eight blue dots indicate the diamonds inside the tracking volume, and the red dots indicate diamonds outside the tracking volume.

The diamond sensors for the CDF BCM system were obtained from Ohio State University. These are the same type of diamond sensors produced for the BCM system for the ATLAS detector at CERN. The sensors cover an area of one $\mathrm{cm}^{2}$ square and are $500 \mu \mathrm{m}$ thick. The wafer from which the diamond was taken was grown by Element Six, Ltd. [1], in collaboration with CERN's RD42 collaboration. The two faces were metalized with an aluminum-based compound developed by Ohio State University specifically for the ATLAS sensors.

The sensor was then set inside a G10 package which provides easy connections to the metalized sides of the sensor. Fig. 2 shows a photograph of the diamond in the G10 package. A new layer of G10 is applied to seal the sensor inside the G10 package, and the package is wrapped in copper to further reduce external electrical noise.

\section{B. Diamond locations}

Overall, 13 diamonds have been installed in the CDF detector. Eight diamond sensors are inside the tracking volume. These sensors operate in a magnetic field of $1.4 \mathrm{~T}$, directed along the beam line, provided by CDF's superconducting solenoid. These diamonds are divided in two groups of four, located in the east and west side of the tracking volume, as shown by the blue dots in Fig. 3. Each group of four is mounted in a support structure at a distance of $1.7 \mathrm{~m}$ from the nominal interaction point (I.P.). The support structure, depicted in Fig. 4, holds the diamonds parallel to the sides of an imaginary square, $4 \mathrm{~cm}$ on a side, through which the beam passes. Thus each diamond is $2 \mathrm{~cm}$ from the beam line. The electric field of these diamonds points towards the beam line, perpendicular to the magnetic field.

Five more diamonds are installed in the outer regions of CDF, where the current BLM system is located. These diamonds are depicted as orange dots in Fig. 3. They are $4.3 \mathrm{~m}$ from the I.P. and about $20 \mathrm{~cm}$ from the beam line. Two diamonds are in the west side and three in the east side. 


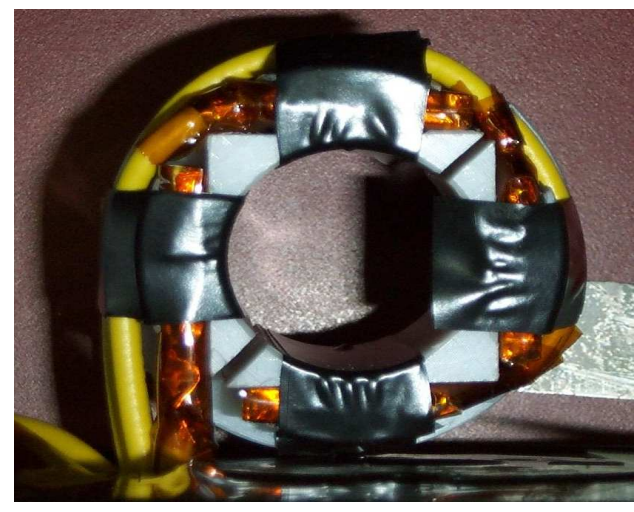

Fig. 4. Diamond support structure holding four diamonds, ready to be installed. The white square with the circular hole is part of the support structure. The beam pipe will pass through the hole after installation. The packaged diamonds are held in place with electric tape.

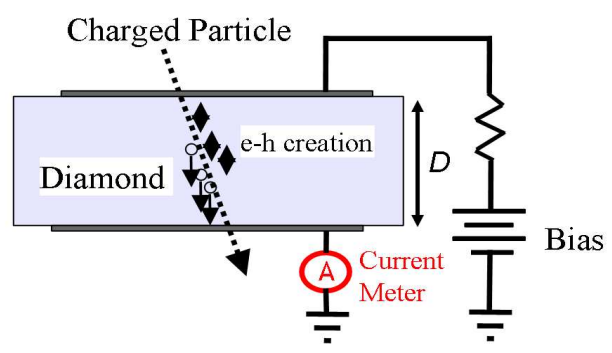

Fig. 5. Schematic of the diamond readout. The current passing through the diamond is measured in the current gauge A. The current is digitized and read out in a custom-modified VME crate, as detailed in the text.

\section{Diamond readout electronics}

The basic principle of the diamond readout is shown in Fig. 5, in which the current passing through the diamond is a measure of the ionizing radiation traversing the diamond. The current measurement is performed using a custom-modified VME crate designed and built by the Electrical Engineering Department of the Fermi National Accelerator Laboratory. This system was designed and built for the Tevatron BLM upgrade project [2], in which the readout electronics of the BLMs in the Main Injector and the Tevatron were upgraded.

The readout electronics integrates every $21 \mu \mathrm{s}$ (one beam revolution), keeping track of the last 65535 measurements. In addition, three sliding sums or buffers are stored, based on the $21 \mu \mathrm{s}$ measurements. These sliding sums are $16 \mathrm{k}, 4 \mathrm{k}$, and $4 \mathrm{k}$ bins deep and hold information from the last $1 \mathrm{~ms}, 50 \mathrm{~ms}$, and $1 \mathrm{~s}$, respectively. The pedestal, or offset, is measured automatically by taking the average of 1024 measurements of the $21 \mu \mathrm{s}$ buffer just before beam injection.

These buffers are dumped to a file upon receipt of a beamabort or end-of-beam signal. The beam-abort signal is issued whenever an anomalous condition is detected in the Tevatron and the beam is discarded immediately to avoid damaging sensitive components in the Tevatron or its detectors. The endof-beam signal is issued when the beam is discarded because its luminosity is too low for useful physics results. In this case the beam is usually replaced shortly with a new beam.

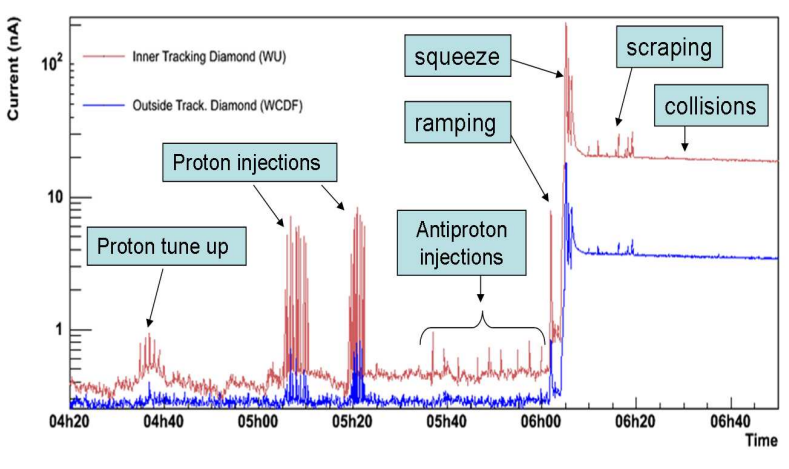

Fig. 6. Current of diamonds during beam injection at the Tevatron versus time. The current of one of the diamonds inside the tracking volume is shown in red. In blue is the current of one of the outside diamonds. Note that the structure is clearly seen for the diamond inside the tracking volume, which is at a radius of $2 \mathrm{~cm}$ from the beam line, while it is less clear for the diamond outside the tracking volume, which is located at a radius of $20 \mathrm{~cm}$.

In each channel the $21 \mu \mathrm{s}$ integration is performed by of two digitizers. While one digitizer collects charge, the other sends its information to be processed, and every $21 \mu \mathrm{s}$ they switch roles.

\section{DIAMOND-BASED BCM DATA}

\section{A. Collected data}

Data has been taken with the diamond system during normal Tevatron operation for nearly a year. Fig. 6 shows the response of two diamonds during the sequence of accelerator events leading to proton-antiproton collisions. One diamond is located $2.0 \mathrm{~cm}$ from the beam inside the tracking volume, while the other is located $20 \mathrm{~cm}$ outside the tracking volume. This figure shows the clear advantage of placing sensors closer to the beam line: the inner sensor can differentiate the structure of all the accelerator events, while the outside sensor cannot resolve them as clearly. While both these diamonds are biased and read out identically, the signal yield of the inner diamond is approximately an order of magnitude larger than the outside one, demonstrating the difference in radiation fields in both locations.

One can also compare the signal yield of a diamond in the outside location to the BLM next to it. Both devices are read out with the same electronics, but while the BLM is biased at $2000 \mathrm{~V}$ the diamond is biased at $500 \mathrm{~V}$. The comparison is made while the Tevatron maintains stable collisions and is plotted as a function of luminosity. In this regime, the remnants of proton-antiproton collisions form the dominant source of radiation. Fig. 7 shows the current for one of the diamonds outside the tracking volume and the BLM next to it. Notice from the figure that for an instantaneous luminosity of about $100 \mathrm{~cm}^{-2} \mathrm{~s}^{-1}$, the BLM yields about $1 \mathrm{nA}$ while the diamond physically next to it yields about $3 \mathrm{nA}$.

On November 9, 2006, a beam-abort signal was issued due to a spark in the separators, and the data of the buffers recorded as shown in Fig. 8. The diamonds and the BLM both report a three-peak structure, but the diamond signals are an order 

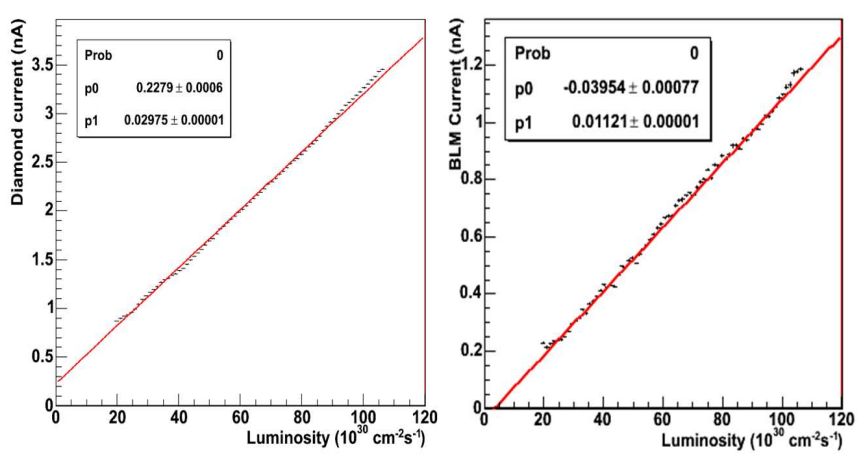

Fig. 7. Current as a function of instantaneous luminosity during stable collisions for the diamond (left) and for the BLM next to the diamond (right).

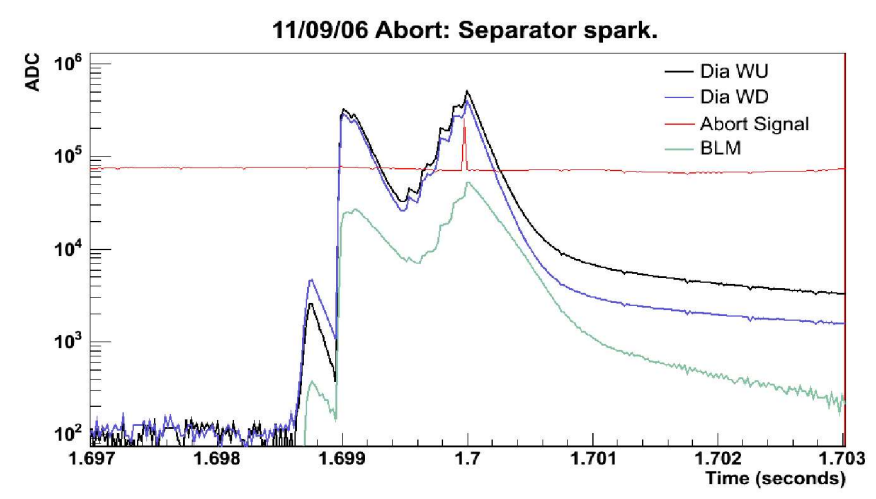

Fig. 8. ADC counts of the $21 \mu \mathrm{s}$ buffer versus time. This information was recorded after a beam-abort signal on November 9, 2006, caused by a spark between electrostatic separators. The figure shows the current in two diamonds (black and blue lines) and a BLM (dark green line). The red line marks the moment of the arrival of the beam-abort signal.

of magnitude larger, and the change in signal for the diamond spans three orders of magnitude. The diamonds saw a big change at least 1 millisecond (about 50 beam revolutions) before the actual beam-abort signal was issued.

Another example of a beam incident is shown in Fig. 9. On March 2, 2007, the Tevatron's parameters were set incorrectly when configuring the collision helix, resulting in high losses that ended in a magnet quench. This plot shows the reading from the four diamonds on the west side, as well as the time the actual beam-abort signal was sent. The tentative abort threshold during this process is 12000 ADC counts; thus, the diamonds would have pulled the abort as much as $500 \mu \mathrm{s}$ (about 25 Tevatron revolutions) earlier than the current system, substantially reducing the risk of incurring damage to the silicon detector.

\section{CONCLUSIONS}

Diamond detectors work well in BCM systems. Diamond sensors are small and can be placed inside the detectors used in high-energy physics experiments. That allows a closer proximity to the beam line and thus a more detailed understanding of the beam structure as shown in Fig. 6. In addition, diamonds

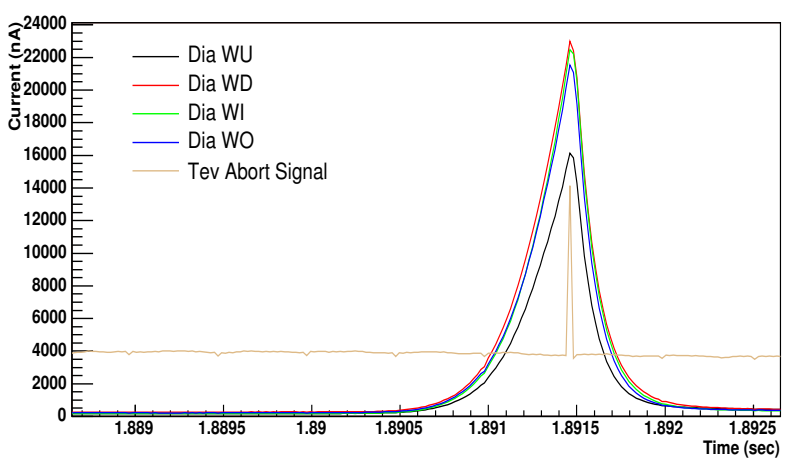

Fig. 9. ADC counts of the $21 \mu \mathrm{s}$ buffer versus time. This information was recorded after a beam-abort signal on March 2, 2007, caused by incorrect parameters when changing to the collision helix. The tentative abort threshold during this time is 12000 ADC counts, which means the diamonds would have pulled the abort as much as $500 \mu$ s before the current system.

showed a larger signal yield than BLMs in the collision regime, as shown in Fig. 7. These features, together with faster electronics, allow for better monitoring of beam conditions in locations closer to radiation sensitive systems in hadron collider detectors.

\section{ACKNOWLEDGMENT}

We would like to thank the CDF technical and scientific staff for their vital contribution, the help of H. Kagan of Ohio State University, and the support of the RD42 collaboration. This work was supported by the CDF-PPD department via the U.S. Department of Energy; the University of California, Los Angeles; the University of Toronto, Canada; and the University of Geneva, Switzerland.

\section{REFERENCES}

[1] Element Six Ltd., King's Ride Park, Ascot, Berkshire SL5 9BP UK

[2] A. Bambaugh, C. Drennan, B. Fellenz, K. Knickerbocker, J. Lewis, S. Pordes, and M. Utes. "BLM Upgrade Users Guide", http://beamdocs.fnal.gov/DocDB/0014/001410/007/BLM 